\title{
Flood Prediction using Artificial Neural Networks: Empirical Evidence from Mauritius as a Case Study
}

\author{
A. Z. Dhunny ${ }^{\mathrm{a}, 1, *}$, R. H. Seebocus ${ }^{\mathrm{b}, 2}$, Z. Allam ${ }^{\mathrm{c}, 3}$, M. Y. Chuttur ${ }^{\mathrm{d}, 4}$, M. Eltahan $^{\mathrm{e}, 5}$, H. Mehta $^{\mathrm{a}, 6}$ \\ ${ }^{a}$ Cyberange Global Holdings PTE, Singapore \\ 68 Circular Road, \#02-01, 049422 Singapore \\ ${ }^{b}$ Department of Physics, Faculty of Science, University of Mauritius \\ $4^{\text {th }}$ floor NAC building, University of Mauritius Reduit MU MU, 80837, Mauritius \\ ${ }^{c}$ Curtin University Sustainability Policy Institute, Curtin University \\ Building 209, Level 1, Room 133, Kent St, Bentley WA 6102 Perth, Australia \\ ${ }^{d}$ Dept. of Software \& Information Systems, Faculty of Information, Communication \& Digital Tech., Univ. of Mauritius \\ $2^{\text {nd }}$ floor Phase II building University of Mauritius Reduit MU MU, 80837, Mauritius \\ ${ }^{e}$ Aerospace Department, Faculty of Engineering, Cairo University \\ 1 Gamaa Street, Giza, EgyptGiza12613 Egypt \\ ${ }^{1}$ zaynah.d@cyberange.io *; ${ }^{2}$ reenahansaseebocus@gmail.com; ${ }^{3}$ zaheerallam@gmail.com; \\ ${ }^{4}$ y.chuttur@uom.ac.mu; ${ }^{5}$ muhammedsamireltahan@gmail.com; ${ }^{6}$ harsh@cyberange.io \\ * corresponding author
}

Article history:

Received 23 July 2020

Revised 26 July 2020

Accepted 9 August 2020

Published online 17 August 2020

Keywords:

Flood prediction

Artificial Neural Networks (ANN)

Microclimate

Mauritius

Case Study
Artificial Neural Networks (ANN) has been well studied for flood prediction. However, there is not enough empirical evidence to generalize ANN applicability to small countries with microclimates prevailing in a small geographical space. In this paper, we focus on the climatic conditions of Mauritius for which we seek to investigate the accuracy of using ANN to predict flooding using locally collected data from 11 meteorological stations spread across the country. The ANN model for flood prediction presented in this work is trained using 20,000 climate data records, collected over a period of two years for Mauritius. Our input climate features are minimum temperature, maximum temperature, rainfall and humidity and our output decision is 'flood' or 'no flood'. Using ANN, we achieved an accuracy of $98 \%$ for flood prediction and hence, we conclude that ANN is indeed a good predictor for flood occurrence even for regions with predominantly microclimatic conditions.

This is an open access article under the CC BY-SA license (https://creativecommons.org/licenses/by-sa/4.0/).

\section{Introduction}

The average temperature of the earth is increasing at an alarming rate and it has been envisaged to increase by a factor of about 1.4 to 5.8 degree Celsius by the year 2100 [1]. An increase in the atmospheric temperature entails the occurrence of many extreme events such as stronger heat waves, formation of intense cyclones, unprecedented flash floods and severe drought events [2] which are set to impact greatly on both the global economy and society. Among the various natural disasters, which affect mankind, flash floods have been reported to cause more casualties in terms of economic loss, death tolls and infrastructural damages. Flooding has become a recurrent phenomenon in the recent decade accounting for about $73 \%$ of damages caused by natural disasters which in turn results in an overall loss of about $\$ 30$ billions [3]. Flash floods are thus a global phenomenon affecting major parts of the world [4][5] as indicated for the year 2018, which marked the occurrence of several deadly flash floods in Kerala, France and Vietnam [6].

In this study, we focus our attention to Mauritius, which is a small island located in the Indian Ocean, off the east coast of Africa and Madagascar. The morphological landscape of Mauritius consists of highlands and coastal regions in a relatively small geographical area of $1865 \mathrm{~km}^{2}$ such that it is typical for the island to experience several microclimates on the same day in different regions. Our study is especially motivated by the occurrence of a series of flash floods in Mauritius 
during past years. The island has been subjected to some major flash flood incidents since the year 2008. These events have become quite recurrent and have caused much infrastructural damage and in some unfortunately occurrences, have led to loss of human lives. For instance, in March 2013, a $152 \mathrm{~mm}$ of rainfall was experienced in Port Louis causing massive flooding that claimed the lives of at least 11 people, besides causing unprecedented chaos and traffic crisis in the city. It is also believed that the recurrent flooding events in several parts of Mauritius can be attributed to the rapid urbanization occurring in various parts of the country, which resulted in the obstruction of actual water evacuation channel beds for constructions purposes [4][5][7]. Given that Mauritius is a developing country and the high likelihood of increased urbanization projects in the future, there is a high need to develop weather forecasting tools that can accurately predict the occurrence of flood.

Weather forecasting is defined as the process of identification and prediction of climatic conditions (e.g., temperature, wind, humidity, etc.) to a certain degree of precision. The results of accurate weather forecasting can be then be used to predict correlated conditions such as flood occurrences. There are different types of forecasting methods such as Native approach, Judgmental approach, Quantitative and Qualitative method, Causal or economic forecasting methods, Time series methods and Artificial Intelligence (AI) methods which can be used for weather prediction [8]. On this, the two most commonly used forecasting methods are statistical methods, which make use of linear data and Artificial Intelligence, which treats nonlinear data.

The use of statistical methods is not a good alternative since weather variables exhibits stochastic behavior and are mostly non-linear in nature [9][10]. AI methods, in contrast, have been extensively used in literature for the modeling of weather forecast using non-linear data [11][12]. Artificial Intelligence algorithms can fall under three main categories: Genetic Algorithm, Neuro-Fuzzy Logic and Neural Networks and each of these can be used as an individual entity for weather forecasting. For the purpose of this study, we will be using the Neural Network category as it caters for the complex nature of weather, as defined by several parameters such as temperature, humidity, rainfall amount, cloud cover, wind speed and associated direction which are all continuously varying on a temporal basis [8]. In fact, Artificial Neural Network has been found to produce results with higher level of accuracy and precision [9]. We give a brief outline of the different works that have found ANN suitable for weather forecasting using the Artificial Neural Network. For a detailed overview on the application of ANN to weather forecast, readers are invited to consult the work of Nayak et al. [13] and Mosavi et al. [14].

Ustaoglu et al. [15] modeled the maximum and minimum temperature in Turkey using three different ANN methods namely the feed-forward back propagation, radial basis function and generalized regression neural network and the linear regression to determine the best model to be used for forecasting. Results obtained from the neural network analysis and the linear regression were compared, and it was found that all the models studied were good predictors for weather forecasting with ANN slightly performing better than the others. ANN was also used to forecast rainfall predictability in the semi-arid Kharosan Province of Iran. The rainfall data used was calculated using the Digital Elevation Model [16] by taking different climatic variables such as Sea Surface Temperature, Sea Level Pressure, and relative humidity which are responsible factors causing the formation of active clouds resulting in rainfall [17]. Here also, results indicated that the ANN model provided fairly good accuracy in predicting rainfall data.

Abhishek et al. [18][19] have also built a prototype ANN model to forecast different weather variables such as temperature, humidity, wind and rainfall. They studied the effect of increasing the number of hidden layers on the result generated by the model, and deduced that by increasing the number of samples and neurons without exceeding an optimum value increases the models precision. Narverkar et al. [6] and Nayak et al. [13] also performed a thorough literature survey on the different algorithm techniques available which can be used with the Neural Network for forecasting purposes. Their analysis concluded that the Multi-Layer Perceptron Network (MLP), BPN, Radial Basis Function Network (RBFN), SOM and SVM are all suitable rainfall predictors.

Hardwinarto [20] used ANN with the Back Propagation Neural Network with three different epochs set. The Mean Square Error (MSE) was used to measure the accuracy of the results, which indicated that the Back Propagation Neural Network produced outputs with greater accuracy. A similar analysis was done by [8], by reviewing the different algorithms namely; Back Propagation Network, Ensemble Neural Network, Artificial Neural Network, Radial Basis Function Network, 
and General Regression Neural Network. Their study concluded in accordance with the work of [20] that the Neural Network with Back Propagation Network algorithm produced results with least errors. Abbhishek et al. [9] further explain that ANN's capability is infallible, even in the case of nonlinear statistics and especially those generated by the weather data; where they are able to perform predictions with minimum error occurrences. Abbhishek et al. [18] also argue that Artificial Intelligence (AI) technologies such as ANN have numerous advantages over traditional methods of weather prediction.

Previous findings therefore suggest that ANN stands as a good candidate for weather prediction compared to other techniques used for weather forecasting. At the same time, it is noted that there is no empirical evidence to suggest that ANN will still have a good level of prediction accuracy in areas where microclimates co-exist, such as in the case of Mauritius. We expect that such evidence will not only add to the literature regarding the suitability of the ANN model for weather prediction, here flood occurrence, but it will also investigate the behavior of the ANN prediction model when real data from regions experiencing microclimates are taken into consideration.

The main goal of this paper therefore is to address the lack of empirical evidence for small geographical regions experiencing microclimates that can be used to support the applicability of AI models for accurate flood prediction. To this end, the following two objectives are set: 1) apply Artificial Neural Network (ANN) to daily climate data (min. and max. temperature, rainfall, humidity) for the small island of Mauritius to develop a flood forecasting model and 2) evaluate the effectiveness of the developed model for flood prediction. The organization of the paper is as follows: section 2 describes the study area and the ANN algorithm for the methodology part, section 3 presents the results and discussions of the results; and conclusions are drawn in section 4 .

\section{Methodology}

The study area for this work is Mauritius. It is a small island forming part of the African Small Islands Developing State (SIDS) network and is situated in the South West Indian Ocean at latitude 20.2 degrees South and longitude 57.3 degrees East. The Mauritian Republic consists of several other islands and about 49 islets surrounding the main island which forms part of the Mauritian sovereignty (Statistics Mauritius, 2013). The main island of Mauritius has a complex topography with a total surface of about $1865 \mathrm{~km}^{2}$. Its orography consists of broken chains of mountainous ranges in the western parts, flat lowlands and a central plateau found at an altitude of about $400-$ $500 \mathrm{~m}$ which represents a former caldera [21]. The island has a tropical maritime climate with two seasons namely summer (varying from November to late April) and winter (varying from June to September), May and October are considered as transition months. The summer season is normally hot and humid with a mean summer temperature of 24.7 degrees Celsius and a higher probability of rainfall while the winter season is cold and dry with a mean temperature of 20.4 degrees Celsius favoring very little rainfall. The summer season, especially the months of January to March accounts for about $40 \%$ of the seasonal rainfall amount due to the southward convergence of the ITCZ towards the subtropical latitudes and the cyclonic activities. Winter season generates little amount of rainfall which can be attributed to anticyclones and active trade winds which bring stable atmospheric air [22].

The total annual rainfall for Mauritius is approximately $2010 \mathrm{~mm}$. This value is subjected to the orographic influence and varies from $1400 \mathrm{~mm}$ in the eastern coastal lowlands to about $4000 \mathrm{~mm}$ on the central plateau and about $800 \mathrm{~mm}$ on the western coasts [23]. The precipitation pattern is modulated on inter annual time scale (greater than one year) in relationship with the influence of large-scale circulation patterns such as the ENSO, IOD and Tropical Cyclones (TC). Given the landscape morphology of Mauritius, it is very common for the country to experience several microclimates in different regions during the same day making weather prediction and consequently flood predictions a challenging task.

As seen in previous studies, ANN is considered one of the most successful machine learning methods that can be used for flood prediction. Here, a prediction model was developed using theano python library to predict flood possibility using Neural Networks with Tanh logistic activation function. The three basic variables constituting the neural network include the set of connecting links, the activation function and the bias. A schematic overview of the ANN architecture used in this study is shown in Figure 1. 


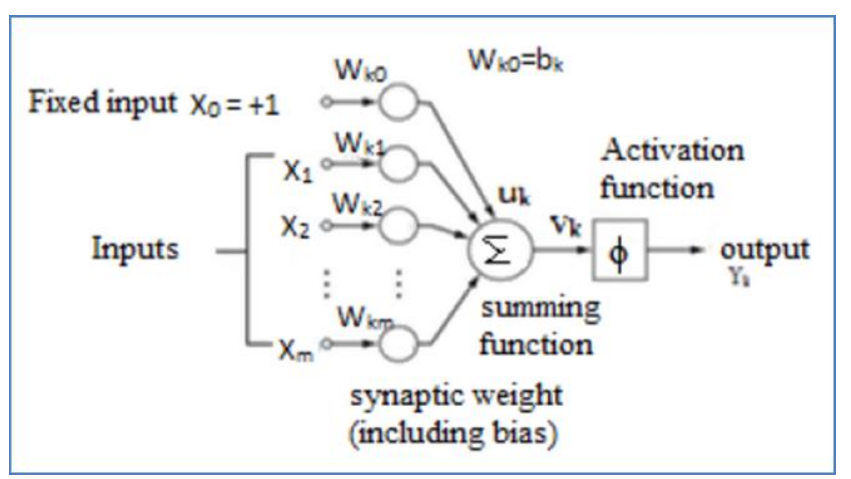

Fig. 1. ANN architecture adapted from [10]

The three layer Neural Network (input, hidden and output layers) can be represented by the mathematical expression given in equation (1) [24].

$$
\hat{Y}_{k}=f_{0}\left[\sum_{j=1}^{n} w_{j i} f_{h}\left(\sum_{j=1}^{m} w_{j i} x_{i}+w_{j b}\right)+w_{k b}\right]
$$

where $\hat{Y}$ is the forecasted $k^{\text {th }}$ output value, $f_{0}$ is the activation function for the output neuron, $n$ is the number of output neurons, $w_{k j}$ is the weight connecting the $j^{\text {th }}$ neuron in the hidden layer and $\mathrm{k}^{\text {th }}$ neuron in the output layer, $f_{h}$ is the activation function for the hidden neuron, $m$ is the number of hidden neurons, $w_{j i}$ is the weight connecting the $i^{\text {th }}$ neuron in the input layer and $j^{\text {th }}$ neuron in the hidden layer, $x_{i}$ is the $i^{\text {th }}$ input variable, $w_{j b}$ is the bias for the $j^{\text {th }}$ hidden neuron and $w_{k b}$ is the bias function for the $k^{\text {th }}$ output neuron.

The input layer represents the data that will be analysed and it is divided into two groups, namely; training dataset to estimate the weight and test dataset to determine the behavior of the ANN model. The input dataset was divided into two parts as follows: 23 months of data used for training and 1 month of data used for testing. The input independent variables or input features of the model were four parameters: rainfall, humidity, minimum temperature, and maximum temperature. These daily data were collected for a time frame two years starting $1^{\text {st }}$ January 2017 to $30^{\text {rd }}$ December 2018 from the Mauritius Meteorological Station (MMS) for 20 stations as indicated by the regions in Figure 2. Out of the 20 stations, data for 11 stations only, was used, as there were missing data from 9 stations. The daily data included the four input features required to train the predictive model. The exact coordinates for each of the $11 \mathrm{MMS}$ locations used to collect input data for the ANN model is given in Table 1.

Feature scaling technique for all of the four input features (rainfall, humidity, minimum temperature, and maximum temperature) was applied to optimize the performance of the ANN algorithm. Feature scaling is done using mean normalization according to equation (2). Moreover, to avoid overfitting the ANN model, extreme data points were removed from our input dataset.

$$
X_{i}=\frac{x_{i}-\min \left(x_{i}\right)}{\max \left(x_{i}\right)-\min \left(x_{i}\right)}
$$

Table 1. List of 11 MMS used for study with exact coordinates

\begin{tabular}{lll}
\hline MMS & Longitude & Latitude \\
\hline Albion & 57.408627 & -20.206125 \\
Baie du Cap & 57.378771 & -20.485812 \\
Belle Mare & 57.777398 & -20.199583 \\
M. Loisir Rouillard & 57.684835 & -20.12059 \\
P. aux Canonniers & 57.56176 & -20.00641 \\
Plaisance (Airport) & 57.678997 & -20.433194 \\
Port Louis & 57.502388 & -20.161998 \\
Providence & 57.621084 & -20.249614 \\
Quatre Bornes & 57.478959 & -20.267172 \\
Rose-Belle & 57.606683 & -20.400288 \\
Vacoas & 57.495288 & -20.291098 \\
\hline
\end{tabular}




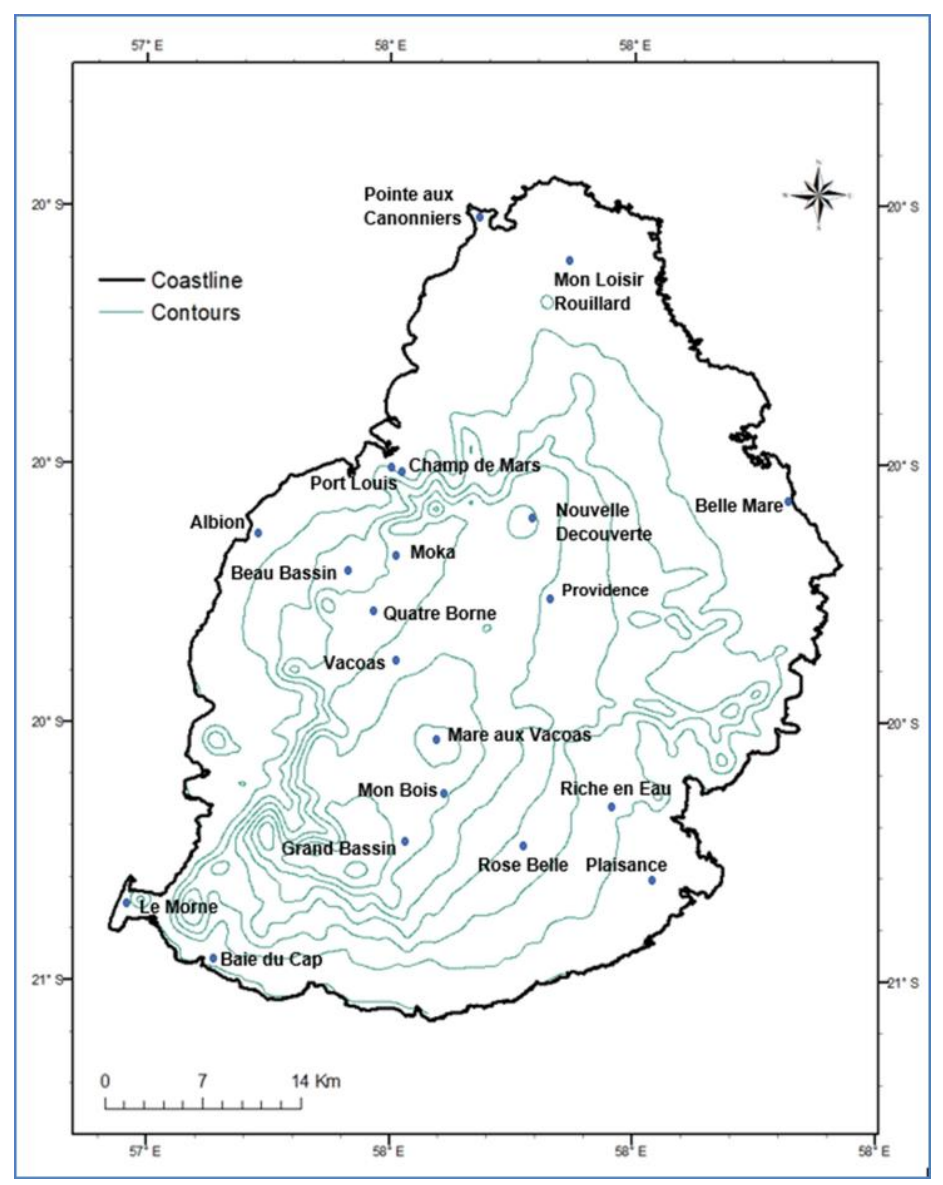

Fig. 2. Geographic distribution of meteorological stations under analysis

The weights calculated by the training dataset are multiplied by each input node value and are then sent to the second layer known as the Hidden layer which can be thought of as an intermediate between the input and the output. The aim of the hidden layer is to determine the underlying complexity in the model, by analysing the variations in the data. At the hidden layer, an activation function is applied before processing the data to the Output layer, where a second activation function is applied to the dataset before generating the final output result [25]. There are different types of activation function such as the log-sigmoid, tan-sigmoid and pure-linear function [18][26][27]. For this study, the logistic Tanh activation function was used for each neuron within the network. The first layer contained 50 neurons and 10 neurons in each hidden layer with the last layer having two outputs for a YES (flood predicted) or a NO (no flood predicted). Once constructed successfully, the ANN model should be able to accurately forecast climatic variables as explained in the work of [18][19]. In this study, we will evaluate the accuracy of our ANN model in predicting flood occurrence using real world microclimatic data collected from different regions.

\section{Results and Discussions}

Time series for the four input features (rainfall, humidity, max. temp. and min. temp.) collected from 11 MMS are shown in Figures 3 to 6 . The data is classified based on the eleven locations selected for this case study (refer to table 1). It is seen that despite the close geographical proximity of the stations, significant variation of climate parameters are recorded confirming the existence of microclimatic conditions across different regions in Mauritius. Brief explanations on each plot are given further.

As for minimum and maximum temperatures, it is noticed that there is a similar trend for all regions spread across Mauritius. The temperature takes a slight peak around January and then drops gradually towards August to again rise to another peak with the cycle repeated again. The temperature graph indicates a sinusoidal trend demonstrating temperature variation over the two 
seasons of Mauritius, (winter and summer). Winter usually occurs around May-October and summer occurs from November to April as demonstrated by Figures 3 and 4. Maximum temperature observed is around 35 degrees Celsius and minimum temperature observed is around 13 degrees Celsius. In contrast to expected increase in temperature [28], there is no noticeable increase in temperature trend for the two years under study for 2017 and 2018 for Mauritius.

In regards to rainfall data collected (Figure 5), we notice a net difference in the amount of rainfall for 2018 compared to 2017 for the same period. In 2017, the month of February was marked with the highest rainfall recorded for regions like Providence, Quatre Bornes and Vacoas, all of which are centrally located on the high lands in Mauritius. Similarly the highest rainfall data was observed for the same regions for the months of March, May, August, September, and December. An exception is noted for the month of November, where the southern region of Mauritius indicated by Rose belle recorded the highest rainfall data compared to the other regions.

In 2018, the beginning of the year was marked by a net increase in rainfall for almost all parts of Mauritius. Recorded rainfall data skyrocketed for the central regions (Providence, Quatre Bornes and Vacoas), while other regions recorded higher rainfall data than for the same period in 2017. In general, it is observed that for the first three months of 2018 (January to March), there was heavy rainfall recorded across almost all the country. A flattening of the plot from April to October is then observed, with another peak of rainfall occurring in December of 2018.

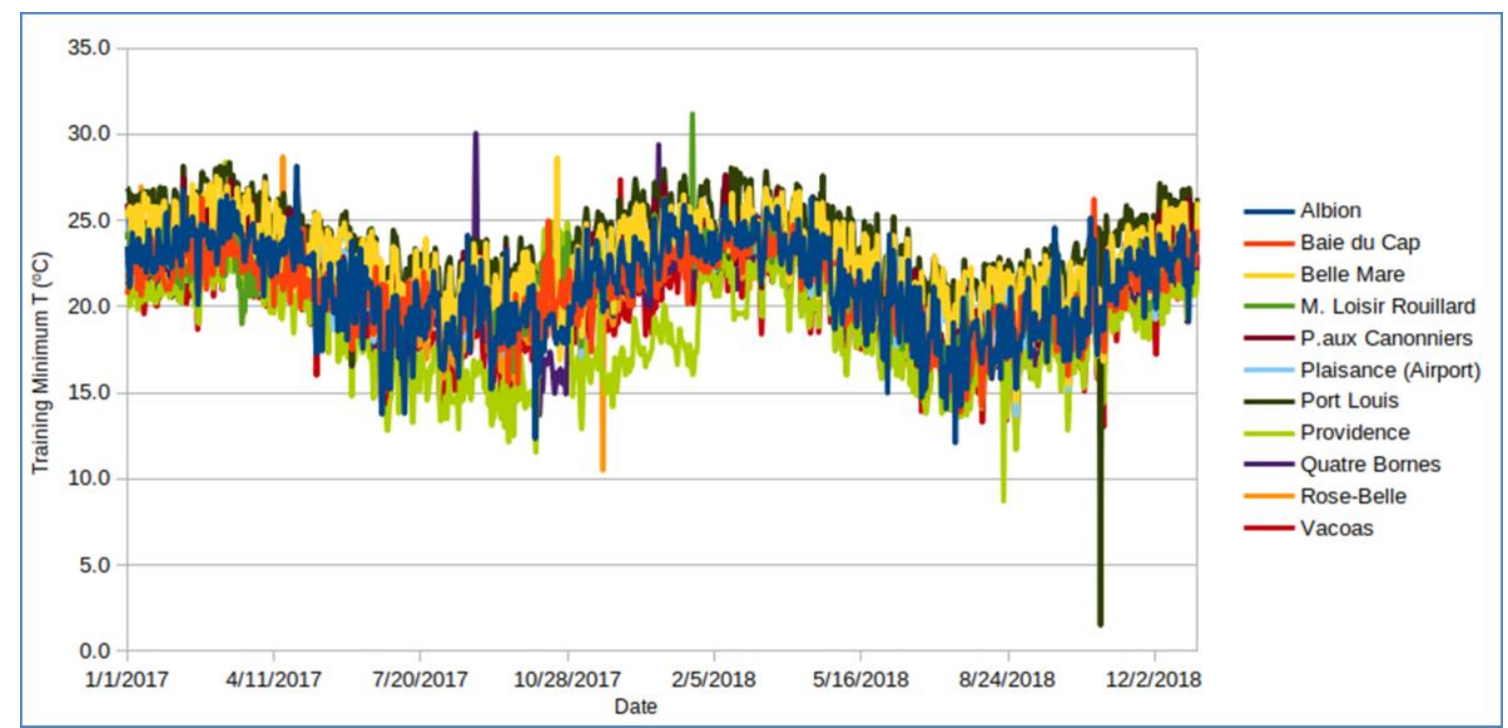

Fig. 3. Time series for minimum temperature

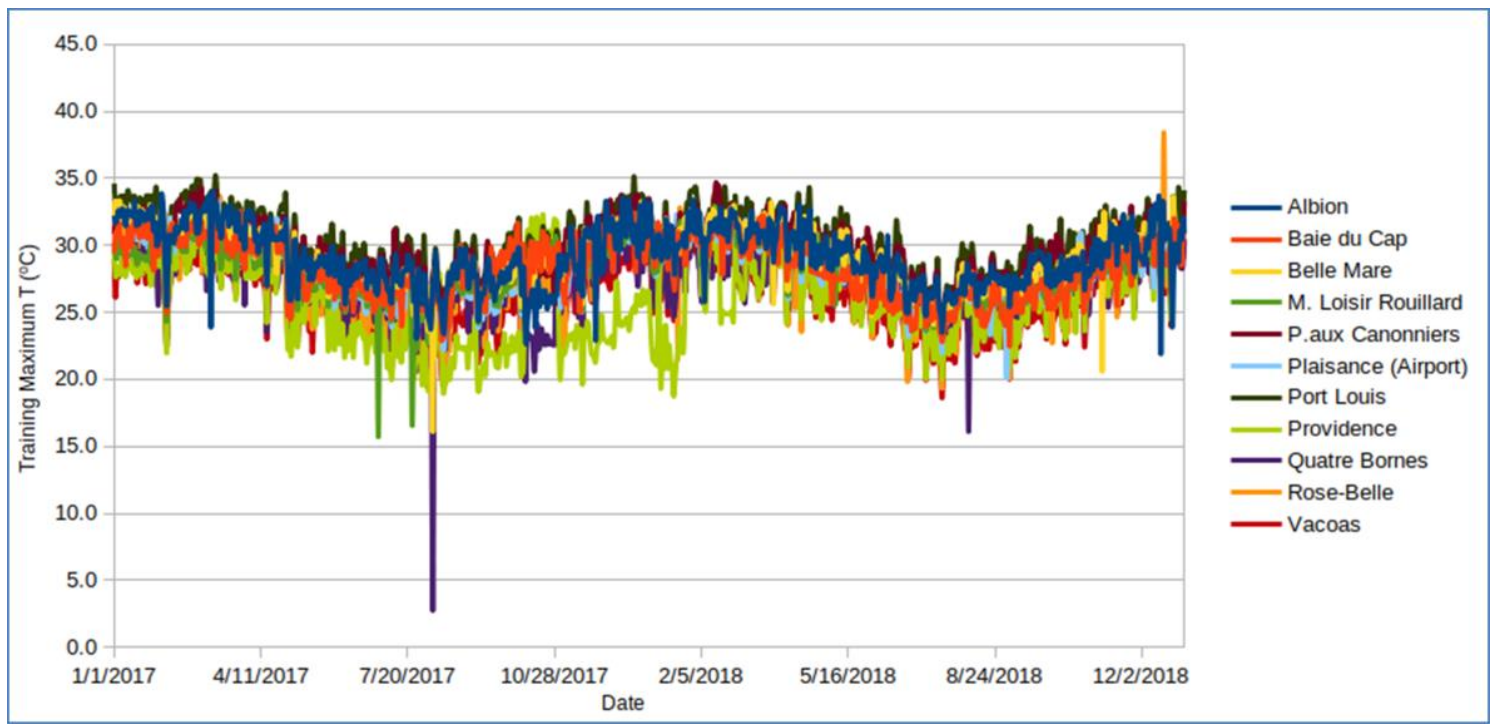

Fig. 4. Time series for maximum temperature 
The trend observed for 2018, thus, is quite different from that of 2017. In fact, it is seen that 2018 is marked by heavier rainfall and for longer periods. At the same time, it is seen that more regions are being subjected to more rainfall than in previous year. This observation contrast with results obtained in [29], whereby rainfall data show a declining trend in southern African region. Such observation highlight the specificity of climatic condition in a region and the need for actual field evidence to better understand environmental phenomena.

As seen in Figure 6, the humidity level varied across the country according to the region (high lands versus low lands). Stations, which were located in high lands (e.g., Providence, Vacoas, Quatre Bornes) registered high humidity compared to stations, which were located near sea level (e.g., Albion, Baie du Cap, Port Louis, P. aux Cannoniers). In some cases, outlier values were noted, but those values were not included in the training data set. The typical observed trend in humidity was also sinusoidal. However, there seems to be no correlation with the season, winter versus summer. Regardless of season or temperature, humidity would have several peaks and troughs all throughout the year. Our results corroborates with observations reported in [30], where the authors found significant variability regarding a best-fit trend in the relative humidity of land, for which further investigation is required.

\section{A. Training the ANN regression model for flood prediction}

The output feature from our data driven model is the decision shown in Figure 7. Based on the different locations, for which climate data was collected, the decision is either [YES=1] which means there is flood or $[\mathrm{NO}=0]$ which means there is no flood for a specific time period for each location.

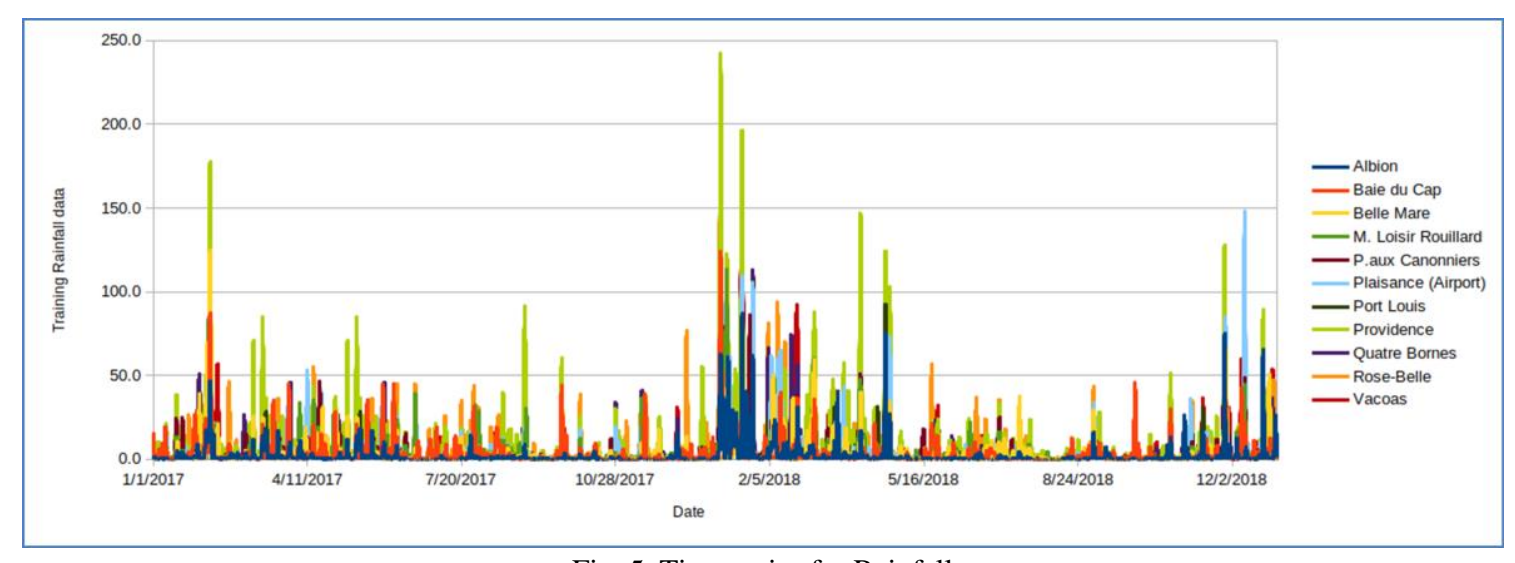

Fig. 5. Time series for Rainfall

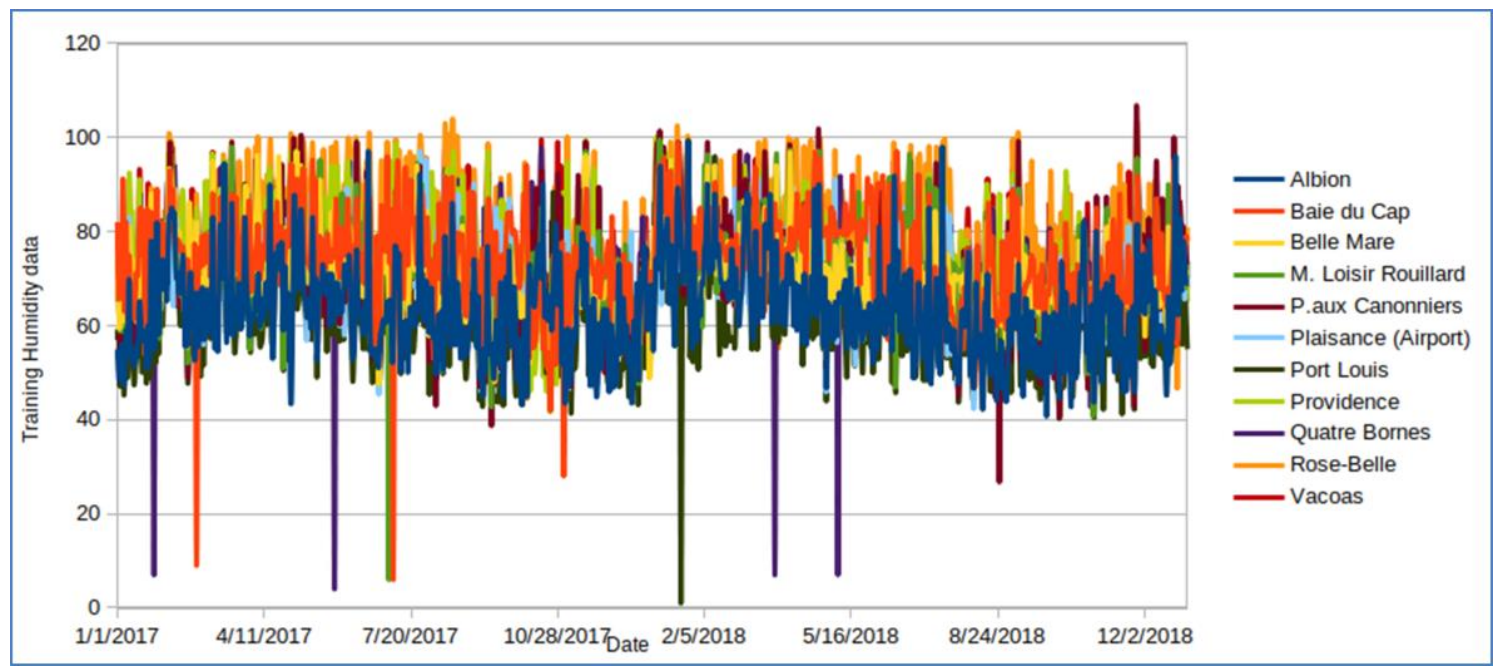

Fig. 6. Time series for Humidity 


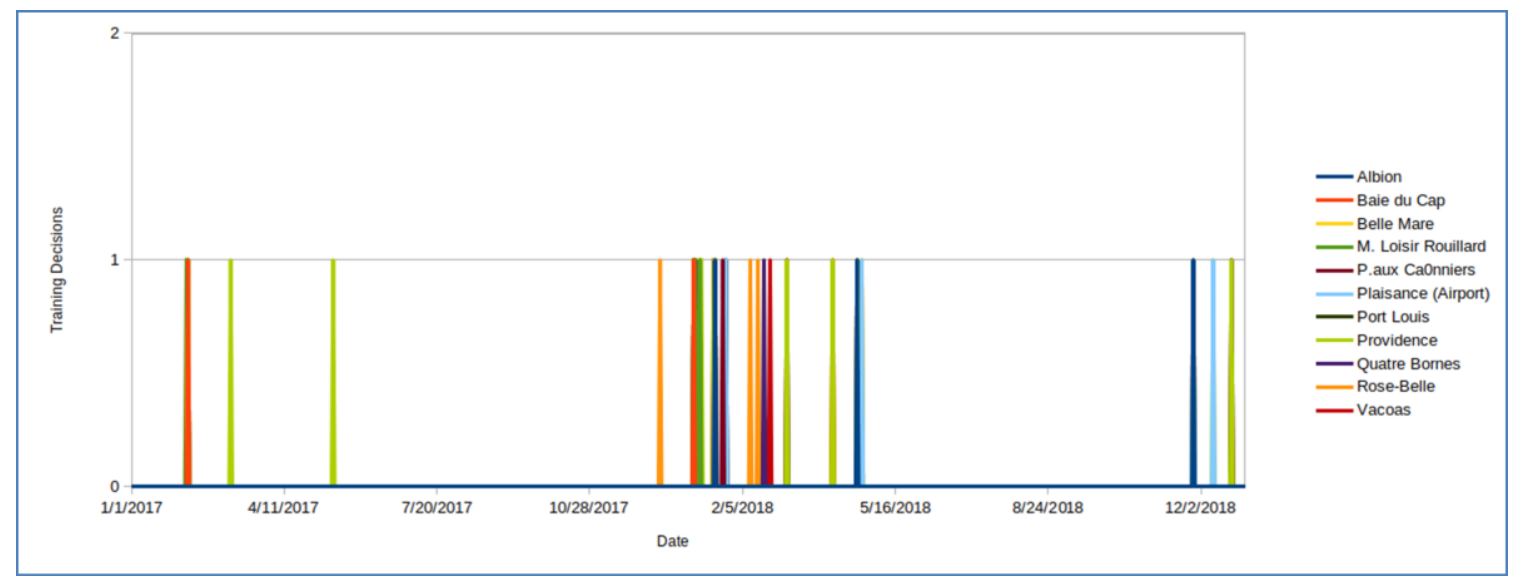

Fig. 7. Time series for output feature decision $([\mathrm{YES}=1] /[\mathrm{NO}=0])$.

As per Figure 7., for the period 1/1/2017 to 12/30/2018, there were several occurrences of flood across different regions of Mauritius as indicated by the colored bars. Those decisions, along with rainfall, maximum and minimum temperature, humidity as input features were fed to our ANN model for training purpose. Our training dataset contained a total of 20,000 input parameters relevant to data collected from the 11 MMS under study for different regions spread over Mauritius. The hypothesis function for the logistic regression is given in Equation (3) and cost function $J(\theta)$, which is named cross entropy (also known as log loss) is shown in Equation (4). Minimization of the cost function was achieved by running Gradient decent algorithm to find the best estimate for the $\theta \mathrm{s}$ parameters.

$$
\begin{aligned}
& \mathrm{h}_{\theta}(X)=\frac{1}{1+\mathrm{e}^{-\theta^{T} \mathrm{X}}} \\
& \mathrm{J}(\theta)=\frac{1}{\mathrm{~m}} \sum_{\mathrm{i}=1}^{\mathrm{m}} \operatorname{Cost}\left(\mathrm{h}_{\theta}\left(\mathrm{x}^{(\mathrm{i})}\right), \mathrm{y}^{(\mathrm{i})}\right) \\
& \operatorname{Cost}\left(\mathrm{h}_{\theta}(\mathrm{x}), \mathrm{y}\right)=-\log \left(\mathrm{h}_{\theta}(\mathrm{x})\right) \text { if } \mathrm{y}=1 \\
& \operatorname{Cost}\left(\mathrm{h}_{\theta}(\mathrm{x}), \mathrm{y}\right)=-\log \left(1-\mathrm{h}_{\theta}(\mathrm{x})\right) \text { if } \mathrm{y}=0
\end{aligned}
$$

The weights for each neuron of the hidden layers were adjusted based on the error produced through backpropagation until the predicted output, as indicated by data fed from decision data for Figure 7., was reached. The error graph, i.e., the value of the cost function at every iteration/loop obtained during the training phase is shown in Figure 8. As illustrated, the error graph converges accordingly and the minimization loops had little effect after around 200 iterations. At this point, we considered, the ANN model to have been optimized in producing appropriate prediction of flooding occurrence based on the input parameters: humidity, temperature and rainfall.

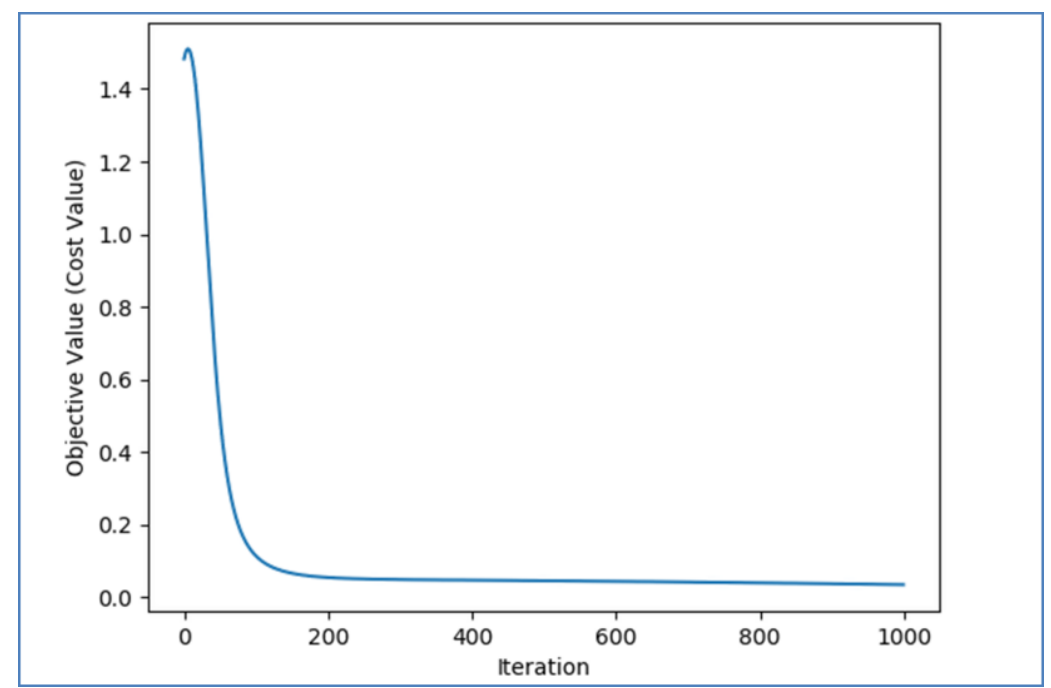

Fig. 8. Cost function for flood data driven logistic regression model 


\section{B. Validating the ANN regression model for flood prediction}

Once our ANN model was trained, we proceeded to testing the accuracy of our model. For the purpose, we used another part of our dataset. To recall, out of the two years of collected data for humidity, min. temperature, max. temperature and rainfall from 11 MMS spread across Mauritius, 23 months of data was used to train our ANN model, and 1 month of data was used for testing purpose. It was ensured that the testing month chosen for testing our model had occurrences of flood in one or more regions in Mauritius. The accuracy of our model was tested using equation (5).

$$
\text { Accuracy }=\frac{T P+T N}{T P+F P+F N+T N}
$$

where TP and TN are the True Positives and True Negatives; FP and FN are the False Positives and False Negatives.

The period of data used for testing and validating the model was $11 / 16 / 2018$ to $12 / 16 / 2018$. Using equation 5, we obtained an accuracy of $98 \%$, which corroborates with findings in [31], where the authors determined that flood prediction using neural networks performed better with higher accuracies than other machine learning algorithms. Our work differ from [31] in that the authors used only temperature and rainfall and achieved an accuracy of 91.185 whereas, here we included humidity as a third input parameter and considered data from different regions exhibiting microclimatic conditions.

\section{Conclusion}

This study applied ANN for flood prediction using daily climate data in Mauritius. The results obtained indicated high-level accuracy in flood prediction, and thus this work adds to the body of literature supporting the application of ANN for flood forecasting. A logistic regression classifier was used as the core algorithm which, processed data collected from 11 meteorological stations data scattered at different altitudes throughout the island of Mauritius. A key contribution of this work is the empirical evidence obtained to support the accuracy of ANN even for regions like Mauritius, which experience microclimatic weather conditions in different regions over the same day. As part of future work on enhancing the efficiency of the ANN model for flood forecasting in Mauritius, further investigations are warranted on the impact of the number of hidden layers, size of dataset and number of regions considered on the performance of the developed model.

\section{Declarations}

Author contribution

All authors contributed equally as the main contributor of this paper. All authors read and approved the final paper.

\section{Funding statement}

This research did not receive any specific grant from funding agencies in the public, commercial, or not-for-profit sectors.

\section{Conflict of interest}

The authors declare no conflict of interest.

Additional information

No additional information is available for this paper.

\section{References}

[1] E. Ashraf, A. Sarwar, M. Junaid, M. B. Baig, H. K. Shurjeel, and R. K. Barrick, "An Assessment of In-service Training Needs for Agricultural Extension Field Staff in the Scenario of Climate Change using Borich Needs Assessment Model.," Sarhad Journal of Agriculture, vol. 36, no. 2, 2020.

[2] Q. H. Nguyen et al., "Land-use dynamics in the Mekong delta: From national policy to livelihood sustainability," Sustainable Development, vol. 28, no. 3, pp. 448-467, 2020.

[3] Y. Zhao, X. Zou, L. Cao, Y. Yao, and G. Fu, "Spatiotemporal variations of potential evapotranspiration and aridity index in relation to influencing factors over Southwest China during 1960-2013," Theoretical and Applied Climatology, vol. 133, no. 3-4, pp. 711-726, 2018.

[4] Z. Allam, "Building a conceptual framework for smarting an existing city in Mauritius: The case of Port Louis," Journal of Biourbanism, vol. 6, no. 1 \& 2, pp. 103-121, 2017. 
[5] Z. Allam and D. Jones, "Promoting resilience, liveability and sustainability through landscape architectural design: A conceptual framework for port louis, mauritius; a small island developing state," in IFLA world congress Singapore, 2018, pp. 1599-1611.

[6] P. Luo et al., "Flood inundation assessment for the Hanoi Central Area, Vietnam under historical and extreme rainfall conditions," Scientific reports, vol. 8, no. 1, pp. 1-11, 2018.

[7] Z. Ahmed, D. R. M. Rao, K. R. M. Reddy, and Y. E. Raj, "Urban Flooding-Case Study of Hyderabad," Global Journal of Engineering, Design and Technology, vol. 2, no. 4, pp. 63-66, 2013.

[8] M. Narvekar and P. Fargose, "Daily weather forecasting using artificial neural network," International Journal of computer applications, vol. 121, no. 22, 2015.

[9] R. Nayak, "Artificial Neural Network Model for Weather Prediction," International Journal of Applied Information System (IJAIS), 2015.

[10] M. P. Darji, V. K. Dabhi, and H. B. Prajapati, "Rainfall forecasting using neural network: A survey," in 2015 international conference on advances in computer engineering and applications, 2015, pp. 706-713.

[11] G. Zhang, B. E. Patuwo, and M. Y. Hu, "Forecasting with artificial neural networks:: The state of the art," International journal of forecasting, vol. 14, no. 1, pp. 35-62, 1998.

[12] A. Tealab, H. Hefny, and A. Badr, "Forecasting of nonlinear time series using ANN," Future Computing and Informatics Journal, vol. 2, no. 1, pp. 39-47, 2017.

[13] D. R. Nayak, A. Mahapatra, and P. Mishra, "A survey on rainfall prediction using artificial neural network," International Journal of Computer Applications, vol. 72, no. 16, 2013.

[14] A. Mosavi, P. Ozturk, and K. Chau, "Flood prediction using machine learning models: Literature review," Water, vol. 10, no. 11, p. 1536, 2018

[15] B. Ustaoglu, H. Cigizoglu, and M. Karaca, "Forecast of daily mean, maximum and minimum temperature time series by three artificial neural network methods," Meteorological Applications: A journal of forecasting, practical applications, training techniques and modelling, vol. 15, no. 4, pp. 431-445, 2008.

[16] N. Dempsey, G. Bramley, S. Power, and C. Brown, "The social dimension of sustainable development: Defining urban social sustainability," Sustainable development, vol. 19, no. 5, pp. 289-300, 2011.

[17] G. A. Fallah-Ghalhary, M. Mousavi-Baygi, and M. Habibi-Nokhandan, "Seasonal rainfall forecasting using artificial neural network," Journal of Applied Sciences, vol. 9, no. 6, pp. 1098-1105, 2009.

[18] K. Abhishek, A. Kumar, R. Ranjan, and S. Kumar, "A rainfall prediction model using artificial neural network," 2012 IEEE Control and System Graduate Research Colloquium, Jul. 2012..

[19] K. Abhishek, M. P. Singh, S. Ghosh, and A. Anand, "Weather forecasting model using artificial neural network," Procedia Technology, vol. 4, pp. 311-318, 2012.

[20] Mislan, Haviluddin, S. Hardwinarto, Sumaryono, and M. Aipassa, "Rainfall Monthly Prediction Based on Artificial Neural Network: A Case Study in Tenggarong Station, East Kalimantan - Indonesia," Procedia Computer Science, vol. 59, pp. 142-151, 2015..

[21] C. G. Staub, F. R. Stevens, and P. R. Waylen, "The geography of rainfall in Mauritius: modelling the relationship between annual and monthly rainfall and landscape characteristics on a small volcanic island," Applied Geography, vol. 54, pp. 222-234, 2014.

[22] C. Mcsweeney, M. New, G. Lizcano, and X. Lu, "The UNDP Climate Change Country Profiles: Improving the accessibility of observed and projected climate information for studies of climate change in developing countries," Bulletin of the American Meteorological Society, vol. 91, no. 2, pp. 157-166, 2010.

[23] D. Senapathi, F. Underwood, E. Black, M. A. Nicoll, and K. Norris, "Evidence for long-term regional changes in precipitation on the East Coast Mountains in Mauritius," International Journal of Climatology, vol. 30, no. 8, pp. 1164-1177, 2010

[24] J. Lee, C.-G. Kim, J. E. Lee, N. W. Kim, and H. Kim, “Application of artificial neural networks to rainfall forecasting in the Geum River basin, Korea," Water, vol. 10, no. 10, p. 1448, 2018.

[25] C. W. Dawson and R. Wilby, "An artificial neural network approach to rainfall-runoff modelling," Hydrological Sciences Journal, vol. 43, no. 1, pp. 47-66, 1998.

[26] I. Maqsood, M. R. Khan, and A. Abraham, “An ensemble of neural networks for weather forecasting," Neural Computing \& Applications, vol. 13, no. 2, pp. 112-122, 2004.

[27] A. Kaur, J. K. Sharma, and S. Agrawal, "Artificial neural networks in forecasting maximum and minimum relative humidity," International Journal of Computer Science and Network Security, vol. 11, no. 5, pp. 197-199, 2011.

[28] C. R. Sunstein, S. Bobadilla-Suarez, S. C. Lazzaro, and T. Sharot, "How people update beliefs about climate change: Good news and bad news," Cornell L. Rev., vol. 102, p. 1431, 2016.

[29] K. Dube and G. Nhamo, "Evidence and impact of climate change on South African national parks. Potential implications for tourism in the Kruger National Park," Environmental Development, vol. 33, p. 100485, 2020.

[30] M. P. Byrne and P. A. O'Gorman, "Trends in continental temperature and humidity directly linked to ocean warming," Proceedings of the National Academy of Sciences, vol. 115, no. 19, pp. 4863-4868, 2018.

[31] S. Sankaranarayanan, M. Prabhakar, S. Satish, P. Jain, A. Ramprasad, and A. Krishnan, "Flood prediction based on weather parameters using deep learning," Journal of Water and Climate Change, 2019. 